\title{
A Preferential Analysis of Physical Properties in Graphene Oxide Doped PEDOT:PSS Hole Transport Layers
}

\author{
Yash Sahoo*, Omprakash Sahoo \\ Department of Physics; University of Massachusetts Lowell, 220 Pawtucket St, Lowell, MA, 01853, USA.
}

\section{A R T ICLEDETAILS}

\section{Article history:}

Received 01 May 2020

Accepted 11 May 2020

Available online 16 May 2020

\section{Keywords:}

Active Layer

Hole Transport Layer

Graphene Oxide

Perovskite

\begin{abstract}
A B S T R A C T
Flexible and semitransparent hole transport layers in perovskite-based photovoltaics have been attracting increasing attention in recent years. Among the benefits they provide, such as being easily reproducible and affordable, they pose their own problems with device degradation, stability, and surface wettability. We probed the graphene oxide doped PEDOT:PSS (GO)/poly(3,4 ethylenedioxythiophene):poly(styrene sulfonate) composite for the application in 2D carbonaceous hole transport layers to improve perovskite solar cells. Advantages and problems are pointed out as well as new perspectives to further modify and retract the drawbacks of conducting polymers. We discussed the advancement of the hole transport layer and described the effects of graphene oxide (dopant) on device transmission, surface wettability, and film quality. Basic principles and mechanisms are covered in the development of polymeric hole transport layers and interfacial properties. Findings included the decrease in device transmission, lessened oxidation of the perovskite, and superior surface wettability. This work highlights the importance and relevance of thin, flexible and powerful photovoltaic systems.
\end{abstract}

\section{Introduction}

Perovskite solar cells (PSCs) have gained increasing attention due to their low cost, decreased thickness, and high mechanical flexibilities [1]. The active layer in our solar cell is methylammonium lead halide $\left(\mathrm{CH}_{3} \mathrm{NH}_{3} \mathrm{PbX}_{3}\right)$ in place of silicon which is common in traditional solar cells. The specific type of PSCs produced implement an inverted planar heterojunction $(p-i-n)$ for the device structure Fig. 1a. The integration of perovskite brings subpar cell performances in comparison to siliconcounterparts. A method to improve the photovoltaic cell performance in the solution processable devices are by implementing a thin film semiconductor known as the hole transport layer. HTLs reduce the disparities in work functions between the anode and perovskite layer. This transparent layer can improve charge carrier mobility and reduce recombination of charges between the HOMO and LUMO of the HTL and perovskite interface. PEDOT:PSS, a well-known conducting polymer and p- type semiconductor used in this experiment poses a host of its own problems including moisture locking, poor surface dispersibility, and mediocre conductivity, however, it boasts unmatched properties and is easily tweaked to achieve favorable results. A major drawback faced during the deposition of HTLs is the formation of pinholes due to lackluster interfacial surface tensions. The presence of pinholes presents a problem where an induced current will short circuit, rendering the device useless. It also decreases the current density and open circuit voltage leading to an overall decrease in the power conversion efficiency of the solar cells. These composite further limits the efficiency of the solar cell due to its hygroscopic nature and inability to block electrons [2]. The goal of maximizing the quantity of charges into the perovskite layer poses the use of dopants to heighten functions of PEDOT:PSS. Recently, consideration has been given to the stable aqueous dispersions of graphene oxide (GO) in addition to PEDOT:PSS to improve the HTL. This nanomaterial promises the ease of processability in water and a large surface area to increase conductivity. Suspensions of graphene oxide flakes have unique structures that account for a mixed presence of $\mathrm{sp}^{2}$ and $\mathrm{sp}^{3}$ hybridizations and high surface areas for electrical current to pass (Fig. 1b). The $\mathrm{sp}^{2}$ hybridization can be credited for (GO)s insulating properties, however, graphene oxide proves to bring superior conductivity than anticipated accredited to electron hopping [3]. Delocalized electrons allow for carrying electric charges. The addition of GO improves stability, and partially removes the hygroscopic PSS dopant from PEDOT, thus improving moisture resistance.

(a)

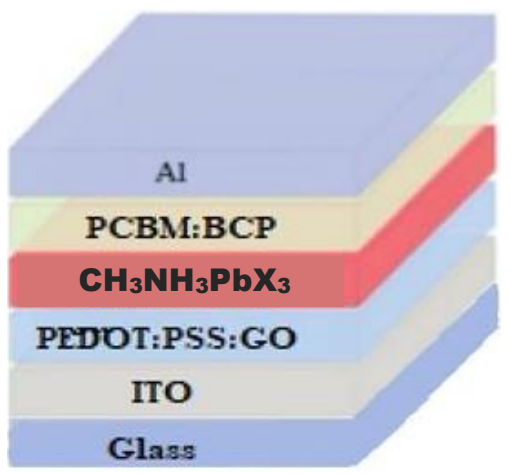

(b)

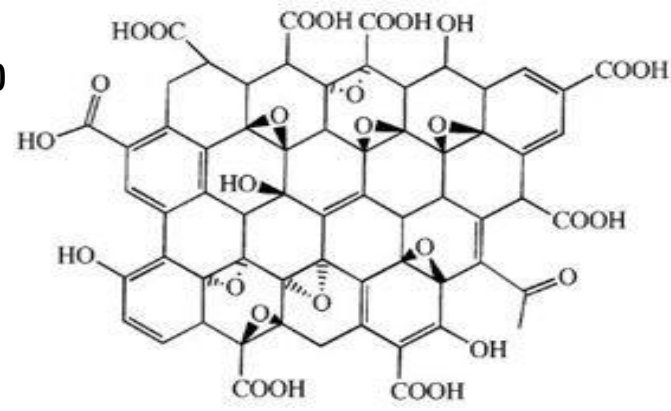

Fig. 1 (a) Device structure which displays the full device and its constituent layers and (b) the structure of graphene oxide, highlighting the functional groups and hybridizations

The amorphous configuration of the conducting polymer can be transformed into highly conductive ones from the separation of the PSS anion. The reduced GO has the potential to improve the wettability of PEDOT:PSS, resulting in better crystallinity and fewer pinholes. The use of thermoelectric polymers such as PEDOT:PSS pose a problem by displaying the lack in conductivity, reigning at $0.2 \mathrm{Scm}^{-1}$ [4]. Adding an organic dopant such as graphene oxide can raise the device conductivity and stability by preventing pinholes. By increasing the wettability of the surface without 
the use of surfactants, a decrease in pinholes will be evident. Furthermore, the acidic PEDOT:PSS can be shielded from the active layer as graphene oxide colloids adsorb to the perovskite interface rather than then PEDOT:PSS itself, increasing long-term stability [5]. In this project, we compare perovskite solar cells with and without the addition of graphene oxide by producing smooth films.

\section{Experimental Methods}

\subsection{Materials}

Graphene oxide flakes define (Sigma-Aldrich, Germany) were purchased and broken down into a fine powder using a mortar and pestle. A $1 \mathrm{mg} / \mathrm{mL}$ dispersion of GO was created by filtering distilled water through a $0.2 \mu \mathrm{m}$ pore filter mixed with graphene oxide powder to create a $1 \mathrm{mg} \mathrm{ml}^{-1}$ dispersion to form a light brown suspension. The solution then underwent an alternating cycle of being homogenized with a vortex and bath sonicator at $40 \mathrm{kHz}$ for 5 minutes each for a total of 2 hours to break the graphene oxide particles into fine colloids.

\subsection{Preparation of (GO)/PEDOT:PSS}

Indium-tin-oxide (ITO) coated glass substrates with sheet resistance of $65 \mathrm{ohms}$ were cut into small squares with dimensions $2.5 \mathrm{~cm} \times 2.5 \mathrm{~cm}$ using a glass cutter. A part ( $2 / 3)$ of the slides were then taped free of air bubbles for the etching process. The substrates were put in a petri dish containing $\mathrm{HCl}$ and zinc powder which were used to etch the slides of ITO, creating an opening for the circuit. The etched ITO slides were then sterilized twice with distilled water and sonicated in soapy water, distilled water, acetone, and isopropanol for 30 minutes for each. The slides were further treated with ultraviolet ozone (PDC-32G) for 20 minutes and later blasted with compressed nitrogen prior to spin coating.

PEDOT:PSS (Al 4083) of a $1.3 \mathrm{wt} . \%$ of PEDOT:PSS in water was used in the preparation of (GO)/PEDOT:PSS. PEDOT:PSS was filtered through a $0.45 \mu \mathrm{m}$ pore filter before added to graphene oxide. The (GO)/PEDOT:PSS dispersion consisted of $0.1 \%$ of graphene oxide by weight. The two composites were mixed together using a vortex and then bath-sonicated for 2 hours.

The control cell with pristine PEDOT:PSS was obtained by spin coating the composite solution at $2000 \mathrm{rpm}$ for 60 seconds [6] and annealing for 15 minutes on a hot plate at $120^{\circ} \mathrm{C}$. This was then set in a vacuum free of heat. The proceeding films were spin coated with (GO)/PEDOT:PSS at $5000 \mathrm{rpm}$ for 45 seconds and then annealed for 20 minutes on a hot plate at $120{ }^{\circ} \mathrm{C}$. The active layer was coated using the following procedures [7]: A $1.2 \mathrm{M}$ solution of lead iodide $\left(\mathrm{PbI}_{2}\right)$ was created using dimethylformamide (DMF) and dimethyl sulfoxide (DMSO) in a 1:9 ratio respectively. The solution was later filtered through a $0.45 \mu \mathrm{m}$ pore filter and left on hot plate for 10 hours at $60{ }^{\circ} \mathrm{C}$. The lead iodide solution was spin coated on the PSC at $4000 \mathrm{rpm}$ for 40 seconds. Later, a $0.42 \mathrm{M}$ solution of methylammonium iodide (MAI) in IPA was made and vortexed. It was then spin coated on top of the lead iodide layer at $4000 \mathrm{rpm}$ for 40 seconds and was then left on the hotplate for 10 minutes. The electron transport layer (ETL) was processed using phenyl- $\mathrm{C}_{61}$-butyric acid methyl ester (PCBM) and bathocuproine (BCP) mixed in a 15\% weight ratio to create $25 \mathrm{mg} / \mathrm{mL}$ of solution using dichlorobenzene. The solution was then spin coated on top of the active layer at $1250 \mathrm{rpm}$ for 40 seconds and was then put on a hotplate for 10 minutes.
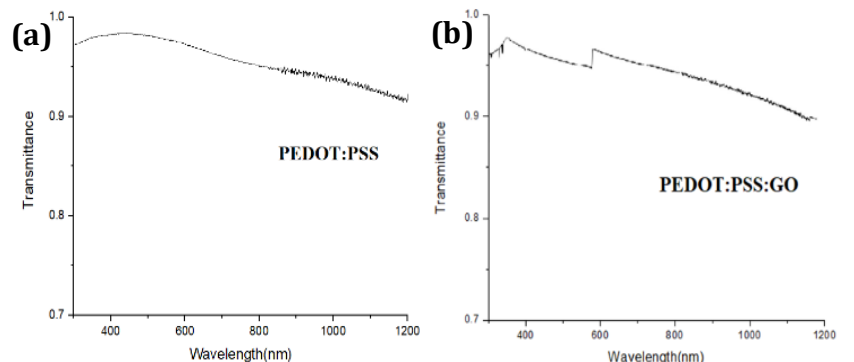

Fig. 2 (a) Transmission of pristine PEDOT:PSS; and (b) Transmission of (GO)/PEDOT:PSS are taken over wavelengths from 300-1200 nm

\section{Results and Discussion}

A spectrometer (Perkin Elmer UV/VIS/NIR) was used to obtain transmission data of PEDOT:PSS and (G0)/PEDOT:PSS from wavelengths of 300-1200 nm. The cells were examined under an optical microscope at $70 \mathrm{x}$ zoom to obtain high resolution images of pinholes and oxidation.
Dispersibility was examined by reflecting light onto the respective coatings of PEDOT:PSS and its doped rendition.

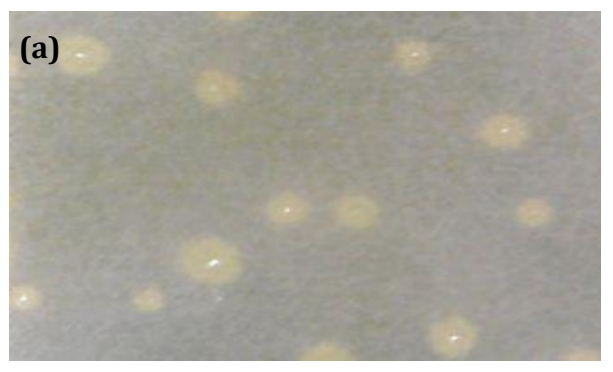

(b)

Fig. 3 (a) Optical image of PEDOT:PSS, here pinholes are displayed by the yellow circles; and (b) optical image of (GO)/PEDOT:PSS, here black streaks are graphene oxide
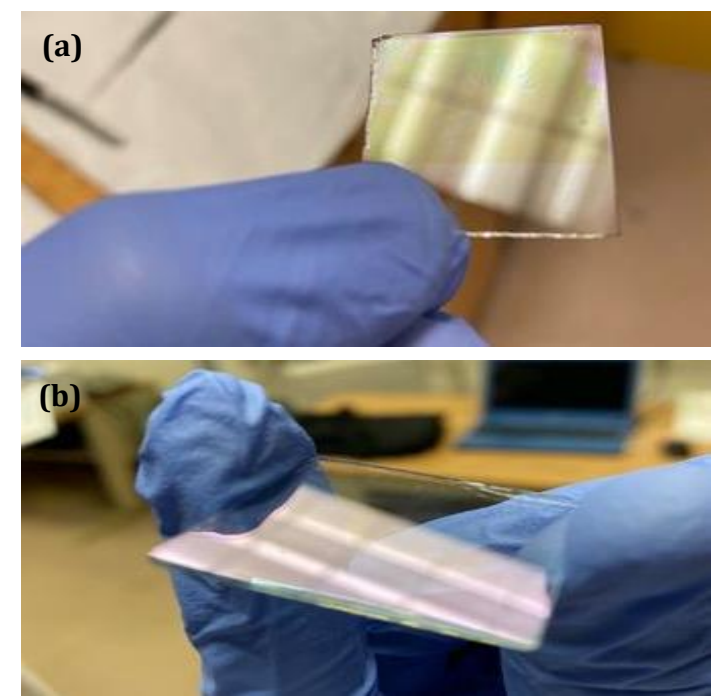

Fig. 4 (a) Image of pristine PEDOT:PSS; and (b) image of (GO)/PEDOT:PSS which are displays spidering and an uneven coating of the solution highlighted by the edges
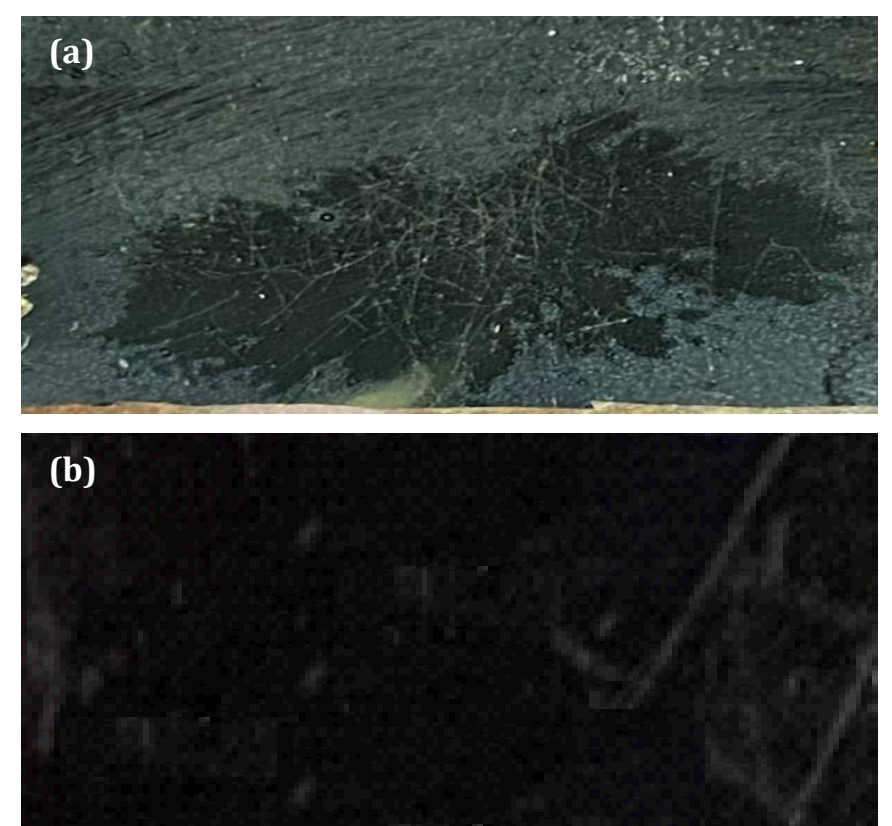

Fig. 5 (a) Optical image of the perovskite in the pristine PEDOT:PSS cell and (b) image of the perovskite in the (GO)/PEDOT:PSS cell. Both cells have oxidation as shown above, a more degraded. 
After spin coating all the respective layers of the both cells exhibited extensive variations in their physical properties. After spin coating of each hole transport layers, the transmission for PEDOT:PSS was slightly higher than that of PEDOT:PSS doped with GO. Fig. 2b displays the steepening transmission of PEDOT:PSS doped with graphene oxide. Images were recorded by an optical microscope at 70x zoom of PEDOT:PSS and (G0)/PEDOT:PSS as shown in Fig. 3a which indicates the presence of pinholes for the control cell with pristine PEDOT:PSS. The hole transport layer with graphene oxide indicates a lack of visible pinholes as seen in Fig. 3b. Fig. 4 depicts the ability of the respective solutions PEDOT:PSS and (GO)/PEDOT:PSS to disperse on ITO. Fig. 4a shows spidering and improper wetting of the surface indicated by the lack of PEDOT:PSS on the edges. Fig. $4 \mathrm{~b}$ displays a surface free of defects. Severe oxidation of the active layer is shown in Fig. 5a. Fig. 5b displays a comparatively less oxidized active layer.

An improvement was seen in the decrease in pinholes in the hole transport layer itself from scattered pinholes to none. This lack of pinholes is connected to the increased surface wettability of the solution, attributed to the modification of the PEDOT:PSS structure. This is due to the partial removal of PSS anions from PEDOT itself caused by the alcohol functional groups in graphene oxide [8].

Slight screening changed the structure and increased the dispersibility of the solution. Reduced $\pi-\pi$ distances brought an expanded volume of PEDOT which attribute the heightened conductivity of the layer. Degradation of the active layer was caused by the oxidation of the perovskite. Graphene oxide served as a barrier between the highly acidic PEDOT:PSS and active layer which minimized the perovskite interface from being oxidized, further increasing device stability [2]. Furthermore, graphene oxide exhibited the recombination of holes and electrons at the interfaces between the HTL and active layer. Electron stability can also be seen in the well-matched work functions between GO (4.89 eV) and PEDOT:PSS (4.95 eV) [9].

\section{Conclusion}

(GO)/PEDOT:PSS was used as the hole transport layer in this PSC to counter the drawbacks of pristine PEDOT:PSS. The addition of the graphene oxide dispersion decreased the overall solution transmission due to graphene oxide colloids absorbing particles of light. Future considerations can be given to the use of sugar alcohols to further screen PSS chains from PEDOT and decrease the hygroscopic nature of PEDOT:PSS. Our results depict that graphene oxide can most definitely be considered as a functional interlayer for solution processable hole transport layers to further develop its application in perovskite solar cells.

\section{Acknowledgement}

Authors Yash Sahoo and Omprakash Sahoo would like to express their deepest gratitude towards Kalpa Henadhiraarachichige and David Campbell for their help and mentorship.

\section{Funding}

This research did not receive any specific grant from funding agencies in the public, commercial, or not-for-profit sectors.

\section{References}

[1] H.S. Dehsari, E. Shalamzari, J. Gavgani, F. A. Taromi, S. Ghanbary, Efficient preparation of ultralarge graphene oxide using a PEDOT:PSS/GO composite layer as hole transport layer in polymer-based optoelectronic devices, RSC Adv. 4 (2014) 55067-55076.

[2] H. Luo, X. Lin, X. Hou, L. Pan, S. Huang, X. Chen, Efficient and air-stable planar perovskite solar cells formed on graphene-oxide-modified PEDOT:PSS hole transport layer, Nano-Micro Lett. 9 (2017) 2150-5551.

[3] G. Eda, G. Fanchini, M. Chhowalla, Large-area ultrathin films of reduced graphene oxide as a transparent and flexible electronic material, Nature Nanotech. 3 (2008) 270-274

[4] Y. Zhimeng, Y. Xia, D. Du, J. Ouyang, PEDOT:PSS films with metallic conductivity through a treatment with common organic solutions of organic salts and their application as a transparent electrode of polymer solar cells, ACS Appl. Mater. Interf. 8 (2016) 11629-11638.

[5] B.Y. Kadem, M. Al-Hashimi, A.S. Hasan, R.G. Kadhim, Y. Rahaq, A.K. Hassan, The effects of the PEDOT:PSS acidity on the performance and stability of P3HT:PCBM-based OSCs, Jour. Mater. Sci.: Mater. Electron. 29 (2018) 19287. 19295.

[6] M. Kumar, S. Rathi, S. Kumar, V. Vyas, A. Singh, Investigation of structure of PEDOT:PSS thin films using X-ray reflectivity, AIP Conf. Proc. 1832 (2017) 080037:1-3.

[7] G. Li, V. Shrotriya, J. Huang, Y. Yao, T. Moriarty, et al., High-efficiency solution processable polymer photovoltaic cells by self-organization of polymer blends, Nature Mater. 4 (2005) 864-868.

[8] X. Fan, W. Nie, H. Tsai, N. Wang, H. Huang, et al., PEDOT:PSS for flexible and stretchable electronics: modifications, strategies, and applications, Adv. Sci. 6 (2019) 1900813:1-41.

[9] J.C. Yu, J.I. Jang, B.R. Lee, G.W. Lee, J.T. Han, M.H. Song, Highly efficient polymerbased optoelectronic devices using PEDOT:PSS and a GO composite layer as a hole transport layer, ACS Appl. Mater. Interf. 6 (2014) 2067-2073. 